\title{
DIABETES
}

\section{Look AHEAD published: weight loss not linked to fewer cardiovascular events in patients with type 2 diabetes}

\author{
$\mathrm{n}$ a multicentre, randomized, \\ controlled study of 5,145 patients \\ who were overweight or obese and
} had type 2 diabetes mellitus, an intensive lifestyle intervention was associated with significant weight loss and improvements in cardiovascular risk factors, but had no effect on the rate of cardiovascular events during $~ 10$-year follow-up. These findings from the Look AHEAD Research Group were published in the New England Journal of Medicine on 24 June 2013.

In their study conducted in 16 clinical centres in the USA, the Look AHEAD Research Group assessed the benefits of an intensive lifestyle intervention involving caloric restriction and increased physical activity in patients who were overweight or obese (mean BMI 36.0) with type 2 diabetes. Enrolled patients (60\% women) were aged $45-75$ years. Patients were randomly allocated either to the intensive lifestyle intervention $(n=2,570)$, with the aim of achieving and maintaining $\geq 7 \%$ weight loss, or to diabetes support and education (the control group; $n=2,575$ ), which involved three group sessions per year for years 1-4, and one group session annually thereafter. Although the trial was not blinded, study-group assignments were not disclosed to researchers assessing clinical characteristics and end points.

At the beginning of the study, the primary end point was defined as the first occurrence of nonfatal myocardial infarction, nonfatal stroke, or death from cardiovascular causes. However, the event rate was lower than expected during the first 2 years of the trial, and so hospitalization for angina was added to the primary end point during the study. In September 2012, the trial sponsors halted the intervention on the basis of a futility analysis and recommendation by the data and safety monitoring board. Median follow-up was 9.6 years.

The intensive lifestyle intervention was associated with a mean weight loss

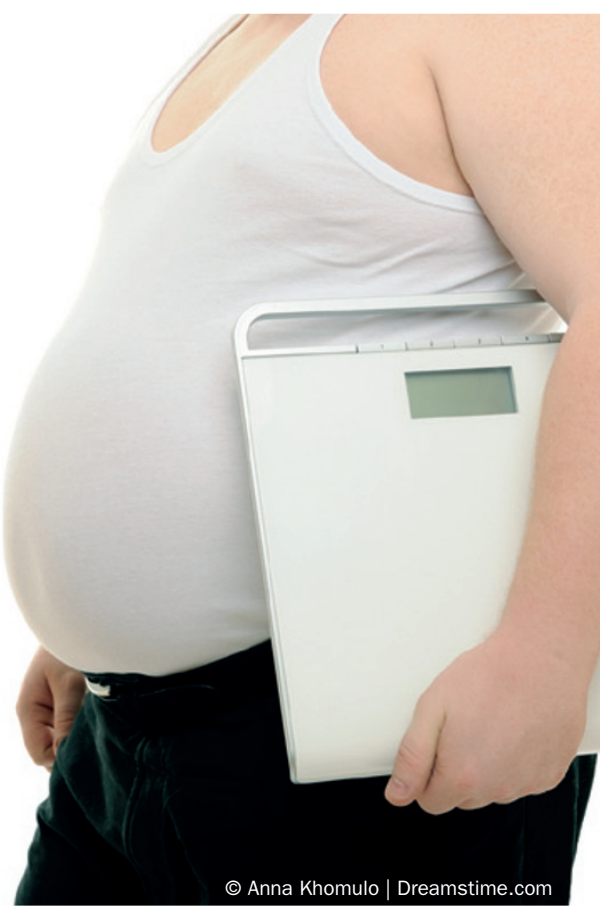

During follow-up, the rate of the revised composite primary end point of nonfatal myocardial infarction, nonfatal stroke, death from cardiovascular causes, or hospitalization for angina was similar for the patients undergoing the intensive lifestyle intervention and those assigned to the control regimen (1.83 events per 100 patient-years versus 1.92 events per 100 patientyears, respectively; HR 0.95, 95\% CI 0.83-1.09, $P=0.51$ ).

In an editorial that accompanied the study report, Dr Hertzel Gerstein highlights that "any between-group difference in [the rate of cardiovascular events] may have been diminished by the reduced use of reportedly cardioprotective drugs ... in the intervention group, possibly because the intervention reduced some cardiovascular risk factors". He also speculates that a modest effect on cardiovascular outcomes might need $>10$ years to become apparent.

The Look AHEAD Research Group remind clinicians that the seemingly neutral effects on cardiovascular outcomes "must be considered in the context of other positive effects observed with this intervention". Such benefits include clinically meaningful improvements in the glycated haemoglobin level, greater likelihood of partial remission of diabetes, increased quality of life, and improved physical functioning and mobility.

Bryony M. Mearns

Original article The Look AHEAD Research Group. Cardiovascular effects of intensive lifestyle intervention in type 2 diabetes. N. Engl. J. Med. doi:10.1056/ NEJMoa1212914

Further reading Després, J.-P. \& Poirier, P. Diabetes: Looking back at Look AHEAD-giving lifestyle a chance. Nat. Rev. Cardiol. 10, 184-186 (2013) 